\title{
Turbulent combustion modelling of a confined premixed methane/air jet flame using tabulated chemistry
}

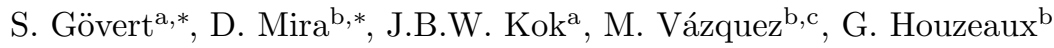 \\ ${ }^{a}$ University of Twente, Faculty of Engineering Technology, Enschede, The Netherlands \\ ${ }^{b}$ Barcelona Supercomputing Center (BSC-CNS), Barcelona, Spain \\ ${ }^{c}$ IIIA-CSIC, Bellaterra, Spain
}

\begin{abstract}
The present work addresses the coupling of a flamelet database, to a low-Mach approximation of the NavierStokes equations using scalar controlling variables. The model is characterized by the chemistry tabulation based on laminar premixed flamelets in combination with an optimal choice of the reaction progress variable, which is determined based on the computational singular perturbation (CSP) method. The formulation of the model focuses on turbulent premixed flames taking into account the effect of heat losses, but it is easily extended to partially premixed and non-premixed regimes. The model is designed for applications in both, Reynolds-averaged Navier-Stokes (RANS) as well as large-eddy simulations (LES) and results for the two methods are compared. A priori analysis of the database is presented to demonstrate the influence of the reaction progress definition and the chemistry tabulation is validated against a one-dimensional premixed laminar flame. The validation of the turbulent case is performed using a turbulent premixed confined jet flame subject to strong heat losses, in which the model shows a good overall performance.
\end{abstract}

Keywords: turbulent combustion modelling, premixed flames, tabulated chemistry, RANS, LES

\section{Introduction}

The increasing energy demand and the yet more restrictive current emissions regulations are forcing the development of more efficient thermal systems and combustion engines 11. As the main part of the global energy supply is still based on fossil fuels [2, the understanding of the combustion process is essential, since the chemical energy of the fuel is converted generally into thermal energy by combustion. Major challenges in the design of modern combustion engines include the reduction of pollutant emissions, increment of fuel flexibility, increasing cycle efficiency and flame stability [3]. To achieve these goals, an accurate description of the interaction of turbulence, chemical reactions and thermodynamics is required. In this context, the use of advanced numerical simulations is becoming a fundamental tool to provide detailed insights into the physical processes at relatively low cost. However, due to the different time and length scales existing in the combustion process, taking into account detailed chemistry in numerical simulations is still a challenge.

Although several attempts have been made to include detailed chemistry in numerical simulations of turbulent flames 4, 5, these applications are still limited to relatively simple geometries and reduced reaction mechanisms due to the high computational cost. In particular, the inclusion of detailed chemistry of complex fuel blends for industrial applications is still prohibitive [6]. To overcome this difficulty, combustion models based on tabulated chemistry have been proposed to investigate the dynamics of turbulent flames at reduced computational cost [7, 8, 9. Instead of solving transport equations for all chemical species involved in the reaction process, one or several scalars are used to represent the combustion chemistry in composition

\footnotetext{
* Corresponding authors

Email addresses: s.govert@utwente.nl (S. Gövert), daniel.mira@bsc.es (D. Mira)
} 
space [10. Several reduction techniques have been proposed to separate fast and slow chemical scales. The intrinsic low-dimensional manifold (ILDM) was developed by Maas and Pope 11] to reduce chemical schemes for certain operating conditions. The CFI model (the abbreviation represents the controlling variables: reaction progress $c$, mixture fraction $f$ and enthalpy scalar $i$ ) developed by Derksen et al. [12] used a similar approach of reducing the chemical subspace by separating the different reaction time scales. The computational singular perturbation (CSP) method [13, 12] was used to identify the species associated to fast and slow scales. These models yield good prediction capabilities in the high temperature range. However, non-equilibrium phenomena associated to the fast time scales, are not correctly reproduced. To overcome this disadvantage, the reduction method was extended to account for low-temperature regions and Lewis number variations by Gicquel et al. 6] in a model called flame prolongation of ILDM (FPI). Another well-established reduction method that is based on the solution of a steady, one-dimensional laminar flame for the generation of a manifold is the flamelet generated manifold (FGM) method proposed by van Oijen et al. [7. These reduction methods have been successfully applied to premixed, non-premixed and partially premixed flames [14, 7, 8, 9, 15, 16, 17.

The combustion model presented in this paper, makes use of the FPI/FGM approach for chemistry tabulation and is based on laminar premixed flamelets. Scalar controlling variables are used to couple the tabulated chemistry to the flow solver in the combustion simulation. In the current framework of premixed, non-adiabatic combustion, a thermo-chemical database is generated by systematically varying the conductive heat losses at the burner inlet using a burner stabilized premixed flame. The database is then parametrized in terms of the normalized enthalpy and the reaction progress variable (RPV), which represents the state of reaction, storing transport properties and the RPV source term.

While the RPV is usually defined based on heuristic approximations and a priori knowledge of the flame characteristics, definitions based on optimization methods have been recently proposed [18, 19. In these approaches, constraints, like monotony and gradient thresholds, are formulated based on the chemical trajectories of the laminar flamelet. Subsequently, optimization tools are used to find the best definition of the RPV, which satisfies the constraints. In the current work, an optimized choice of the RPV definition is proposed making use of the computational singular perturbation (CSP) method [13, 12. The CSP method is applied to the laminar premixed flame computation prior to the tabulation process in order to obtain a RPV definition, in which the information about the different chemical time scales is incorporated. This results in an optimized choice of the RPV yielding to a more uniform distribution of the chemical subspace over the entire range and a reduction of the species derivatives in the thermo-chemical database. Furthermore, no a priori knowledge of the flame characteristics is required for the RPV definition.

An approach based on presumed shape probability density functions (PDF) is applied for chemistryturbulence interaction. The proposed model is applied to address high-fidelity numerical simulations in the context of large-eddy simulation (LES), but is also designed to provide acceptable results for industrialtype applications for Reynolds-averaged Navier-Stokes (RANS) simulations. Therefore, in the course of the paper, the model definition is discussed with a view to the differences between RANS and LES, and finally the results of the two formulations are compared for a premixed turbulent jet flame.

The proposed combustion model is implemented in the High-Performance Computing (HPC) multiphysics code Alya 20. Alya is based on the Finite Element method using the Variational Multiscale Stabilization (VMS) approach [21] and is designed for large-scale parallel applications [22]. Even though the current work focuses on premixed combustion and the influence of heat losses, the model formulation can be extended to partially premixed and diffusion flames by the addition of the mixture fraction as an additional controlling variable. A special computation of the temperature based on a polynomial expression is used, which allows the application of the model in a low-Mach framework as well as for fully compressible flows.

In the current paper, the proposed combustion model and the implementation in the HPC finite element code Alya is validated for the turbulent premixed jet flame that has been experimentally investigated by Lammel et al. 23. The conditions under investigation correspond to a confined lean premixed methane/air flame with equivalence ratio of 0.71 . The burner is operated at ambient pressure with a mixture preheated up to $573 \mathrm{~K}$. The same test case has been used for numerical validation by other authors and the main results are subsequently summarized. Donini et al. 24] investigated the influence of heat loss using a FGM implementation in the commercial CFD code CFX in combination with a RANS approach. It was shown 
that for the current test case the addition of heat losses in the combustion model is essential to correctly predict the flame structure. Fancello et al. 25] performed a LES simulation of the premixed jet flame using the FGM model in OpenFOAM and Proch et al. [26] used the test case to validate different heat loss modelling approaches for FGM in the context of LES. The present work is a contribution to the modelling of the combustion dynamics of this test case, in which RANS and LES are systematically compared using the same turbulent combustion model and numerical methods.

The current paper is organized as follows. In section 2.1, a detailed description of the chemical database calculation is given followed by the mathematical modelling employed for turbulent calculations in RANS and LES (section 2.2 and 2.3). In a first testing step, the formulation of the combustion model is compared against a detailed chemistry computation for a one-dimensional laminar premixed flame in section 3 . Subsequently, in section 4 the model performance for RANS and LES is evaluated using a turbulent premixed jet flame subjected to heat losses.

\section{Mathematical modelling}

This section describes the mathematical modelling used in the current work to obtain the solution fields for RANS and LES. The proposed combustion model is described in detail. Focus is set on the procedure of the chemistry tabulation and the transport equations that are used to represent the combustion chemistry in the numerical calculation. Moreover, the utilized governing equations of fluid dynamics are presented in the low-Mach framework with emphasis on the differences between RANS and LES.

\subsection{Chemistry tabulation}

The proposed combustion model is based on the generation of a thermo-chemical database from a detailed chemistry calculation of a one-dimensional premixed flame. This procedure reduces the stiffness of the reacting flow simulation and is performed in a pre-processing step. In this section, the tabulation procedure is described. First, the solution procedure of laminar premixed flame calculations is introduced, which serves as a base for the chemistry tabulation. Subsequently, the reaction progress variable is defined based on the CSP method and the influence of this definition on the tabulation is discussed. Finally, the addition of heat losses in the thermo-chemical database as well as the the treatment of turbulence-chemistry interaction are presented.

\subsubsection{One-dimensional laminar premixed flame}

The laminar premixed flame calculation is based on the solution of a freely propagating laminar flame using detailed chemical kinetics and transport properties. The conservation equations of mass, energy and species mass fractions along with the equation of state for an ideal gas mixture are solved for a steady, isobaric, quasi-one-dimensional flame propagation problem. The computation is carried out using PREMIX [27], which is part of the CHEMKIN library [28]. The GRI-Mech 3.0 detailed mechanism [29] with 53 reactive species and 325 elementary reactions is used. An additional transport model has been added to the original source code that makes use of the unity Lewis number assumption. Using this assumption, the Lewis number is considered to be unity for all species and constant throughout the flame. Thereby, the complexity of the modelling is significantly reduced at the cost of neglecting the minor effects of differential diffusion. As recently demonstrated by Donini et al. 30, the influence of differential diffusion in the modelling of laminar methane flames is negligibly small. In a turbulent framework, the diffusion processes are governed by turbulent diffusion and the influence of the Lewis number assumption is even lower.

In the thermo-chemical database generation process, a freely propagating flame is used for the adiabatic case, while a burner stabilized flame formulation is employed to take into account heat losses in the chemistry tabulation (see section 2.1.3. The general solution of a one-dimensional laminar premixed flame calculation is presented in Fig. 1a showing the principal evolution of reactant, product and intermediate species along the spatial coordinate $x$. The reactants are consumed by chemical reactions to intermediate species and radicals. From these intermediates the combustion products are formed. 


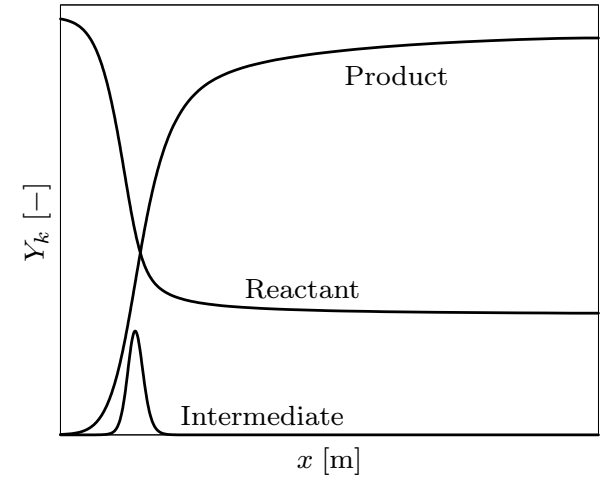

(a) Cartesian space

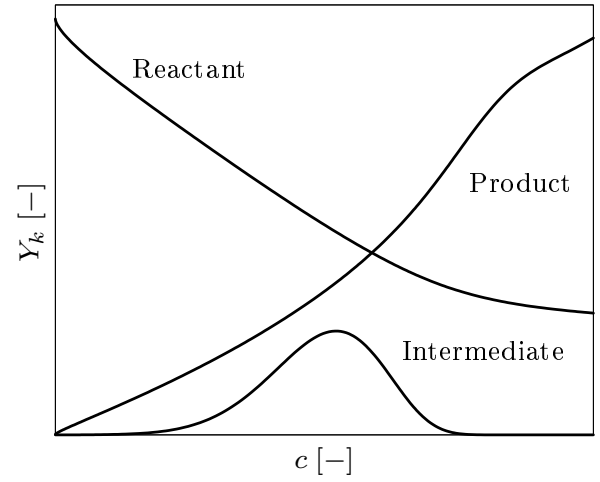

(b) Reaction progress space

Figure 1: General solution of a one-dimensional laminar premixed flame calculation.

\subsubsection{Reaction progress variable definition}

The proposed model is based on the flamelet concept, in which the flame is composed by a set of onedimensional flamelets. This modelling approach is based on the assumption that the local flame structure in a multi-dimensional problem does not differ from the one-dimensional case. In order to use the flamelet description for a multi-dimensional flame calculation, the laminar premixed flamelet is transferred to the reaction progress space. The formulation requires the definition of a reaction progress variable $c$, which represents the evolution of the combustion process from unburnt to burnt mixture. The definition of $c$ is typically based on the the composed species mass fraction $\eta[31,32,33$, which is also referred to as the unscaled progress variable:

$$
\eta=\sum_{k=1}^{K} b_{k} Y_{k}
$$

where $Y_{k}$ is the mass fraction of species $k$ and $K$ is the total number of species in the reaction mechanism. $b_{k}$ describes the composition of the reaction progress variable and can be understood as a weight factor indicating the contribution of the mass fraction of species $k$ to the composed mass fraction $\eta$. The composed species mass fraction is determined for all points in between the limits of unburnt reactants and the fully burnt mixture. These two conditions are then used to normalize the RPV:

$$
c=\frac{\eta-\eta^{u}}{\eta^{b}-\eta^{u}}
$$

where the superscripts $u$ and $b$ indicate the unburnt and burnt composition, respectively. Consequently, if $\eta$ is monotonic between these two points, the reaction progress is zero in the limit of unburnt gases and unity for the fully burnt mixture. The normalized formulation will be referred to as reaction progress variable in the course of the paper. In Fig. 1b, the result of the transformation from physical space to composition space is illustrated for the general case of a laminar premixed flame solution.

The $b$-vector, which describes the contribution of each species $k$ to the composed species mass fraction $\eta$ can be defined by any linear combination that ensures a monotonic behaviour of $\eta$ along the entire flamelet. Different definitions have been proposed in the literature usually based on heuristic considerations and varying with the applications and fuel type [7, 8, 9, 15. In the current work, a new method for the determination of the $b$-vector is proposed based on the Computational Singular Perturbation (CSP) method. The application of this method in turbulent combustion modelling has its origin in the definition of reduced chemistry models. The CSP mechanism is applied to identify the species associated with the fastest chemical time scales. By removing these scales, a slowly developing low-dimensional chemical manifold is constructed [13, 12]. In the current case, the CSP method is applied to obtain a $b$-vector in which the information about the different time scales is incorporated. This results in an optimized choice of the RPV 
yielding to a more uniform distribution of the chemical subspace over the entire RPV range and a reduction of the species derivatives in the thermo-chemical database. The use of the CSP reduces the uncertainty associated to the definition of the RPV and ensures an optimize choice for each application.

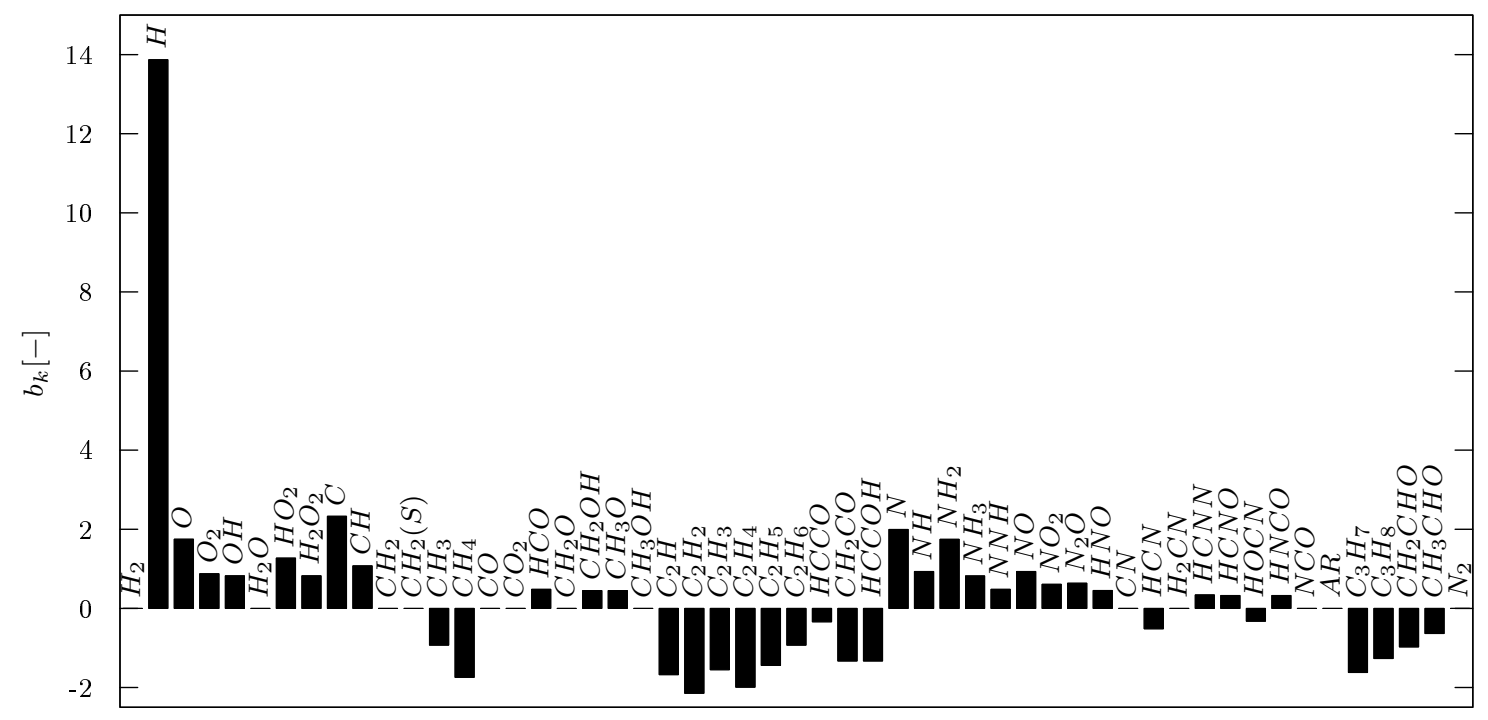

Figure 2: Visualization of the $b$-vector determined by CSP.

As the $b$-vector is computed by the CSP method based on mole fractions, a transformation is required to obtain the $b$-vector for mass fractions prior to the use in Eqs. (1) and (2) using the relation:

$$
b_{k}=b_{k}^{x} \frac{W}{W_{k}}
$$

where $b_{k}^{x}$ is the coefficient based on mole fractions and $b_{k}$ is the corresponding coefficient based on mass fractions. This correction is especially important for species with a molecular weight different from the mean molecular weight of the mixture $W$. The value of the mean molecular weight of the mixture varies slightly with the progress of chemical reaction. To obtain a constant $b$-vector, the mean molecular weight used for this operation is assumed to be constant along the flamelet and is calculated based on the reactants. Due to the very small variation of the mean molecular weight, the influence of this assumption is negligible.

In Fig. 2, the resulting b-vector is presented, showing the contribution of the different species to the composed species mass fraction. The effect of the CSP-based reaction progress variable definition is visualized in Fig. 3 based on the RPV source term. The CSP-based definition is compared to other possible closures, which are frequently used in the framework of flamelet-type combustion models and represent linear combinations of combustion products, reactants or intermediate species. The selected combinations used for comparison correspond to common closures for methane and are given by:

$$
\begin{aligned}
& \text { 1) } b_{\mathrm{CO}_{2}}=1, b_{\mathrm{CO}}=1 \\
& \text { 2) } b_{\mathrm{CO}_{2}}=1, b_{\mathrm{CO}}=1, b_{\mathrm{H}_{2} \mathrm{O}}=1, b_{\mathrm{H}_{2}}=1 \\
& \text { 3) } b_{\mathrm{O}_{2}}=-1
\end{aligned}
$$

All the aforementioned definitions result in a similar shape of the RPV source term. The source term based on the CSP mechanism, on the other hand, features some significant differences coming from the fact that the reaction time scales are taken into account in the RPV definition. In comparison to the reference definitions, the maximum source value is lowered by about 30\%. Furthermore, the location of the peak value is shifted to a lower RPV value, which results in a more uniform distribution of the chemical reaction 
activity over the entire reaction progress space. This differences have a positive effect on the gradients that occur in the RPV space. Lower gradients are beneficial for the discrete PDF-integration procedure that is used for the treatment of turbulence-chemistry interactions (see section 2.1.4), as the absolute difference in each integration interval is reduced. In addition, it lowers the required database density to accurately resolve the chemistry in the complete numerical simulation. In the current case, the RPV space in the database is discretized with 100 points with a linear subdivision. Due to the different initial slope of the RPV source term, the definition might as well be beneficial for other applications like the prediction of low temperature phenomena and (auto-)ignition. The use of CSP for this kind of applications will be analysed separately in a consecutive paper.

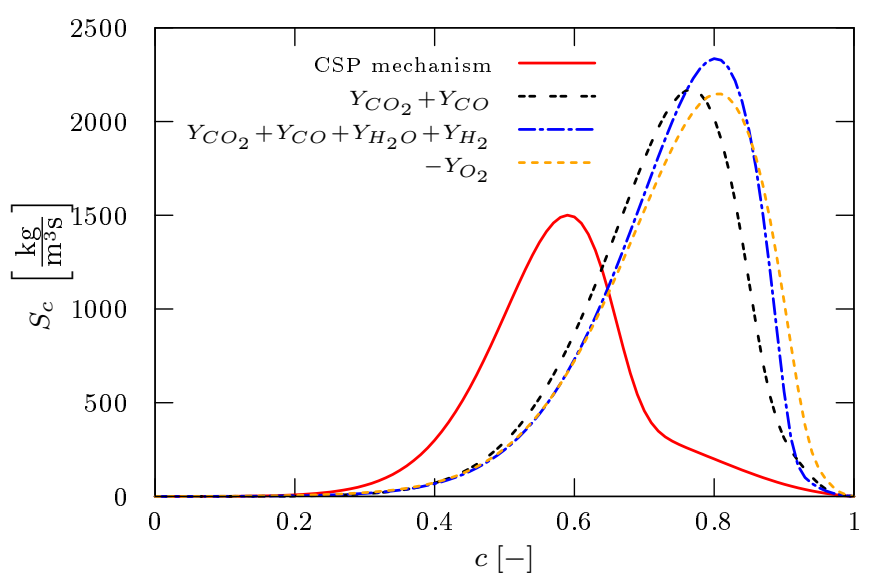

Figure 3: Comparison of the RPV source term for different reaction progress variable definitions.

\subsubsection{Heat loss inclusion}

For cases with low heat losses and/or compact flames, the influence of the heat loss on the chemical reaction rate is minor and can be neglected [9]. However, due to the strong temperature dependency of the chemical reaction rates, special treatment is required for cases in which the flame can interact with or gets close to the walls. In general, the heat losses lead to a reduction of the local temperature and, hence, an increase of the chemical reaction time scales.

For such cases, several flamelets at different enthalpy levels are required in order to accurately describe the combustion process [34. Two different approaches can be chosen for the creation of the flamelets at reduced enthalpy: either the inlet temperature is reduced using a freely propagating flame configuration or the conductive heat losses at the burner inlet are increased by a reduced mass flow rate using a burner stabilized calculation. Fiorina et al. 35] have shown that both approaches lead to identical results in reaction progress space, but as the second approach has a lower limit at which a solution can still be obtained, it is generally preferred and used in this work.

The different enthalpy levels introduce a new dimension in the chemical manifold and the normalized enthalpy scalar is used as an additional controlling variable:

$$
i=\frac{h_{\min }-h}{h_{\min }-h_{\max }}
$$

Consequently, a unity enthalpy scalar corresponds to adiabatic conditions, while a value of zero refers to the maximum heat loss that is incorporated in the chemistry tabulation. The maximum value of the enthalpy $h_{\max }$ is obtained from adiabatic conditions and the associated mass flow rate is obtained from a freely propagating flame case. The lower limit $h_{\min }$ is determined based on the source term of the reaction progress. Laminar premixed flamelets with increased heat losses are computed until the reaction source term vanishes. For the current case, the laminar flame speed of the flamelet with the highest heat losses is 
reduced to about 3 percent of the maximum flame speed. In a practical combustion problem, the heat losses can be higher than the lower limit provided in the chemistry tabulation. However, as can be extracted from Fig. 4. the reaction source term vanishes at the lower tabulation limit. Therefore, additional heat losses still affect the local temperature but do not contribute to any further change of the chemical composition. Due to this selection approach for the lower limit of the enthalpy normalization, the number of required entries in the database is reduced.

In between the two limits, several flamelets are obtained to ensure a sufficient resolution of the thermochemical database. For the current case, the enthalpy scalar is tabulated at 40 discrete values with a linear subdivision. The resulting two-dimensional database is visualized in Fig. 4 on basis of the source term of the reaction progress variable, which allows an analysis of the effect of heat losses on the chemical reactions. The absolute value of the source term is reduced with increasing heat loss, slowing down the overall chemical reaction rate. Additionally, the peak value of the source term is shifted to higher values of the reaction progress variable.

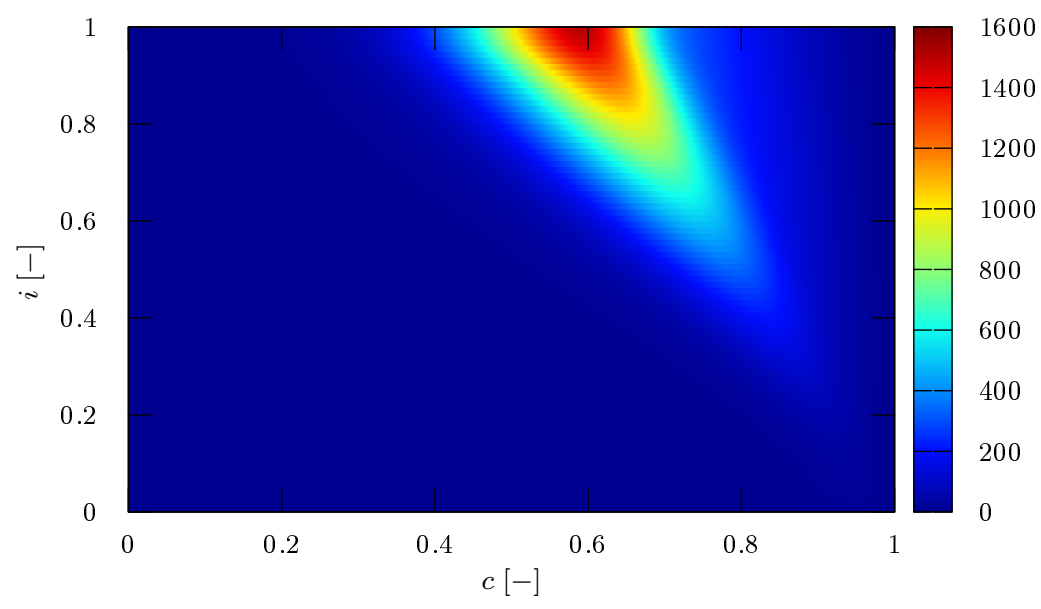

Figure 4: Source term of reaction progress variable $S_{c}\left[\frac{\mathrm{kg}}{\mathrm{m}^{3} \mathrm{~s}}\right]$ for non-adiabatic case.

\subsubsection{Turbulence treatment}

To account for turbulence-chemistry interactions, a stochastic approach based on a presumed-shape probability density function (PDF) is employed. A Favre-averaged description of the governing equations is followed to avoid the modelling of terms including density fluctuations [36]. Assuming an adiabatic calculation, the chemistry depends only on the reaction progress variable, so the ensemble Favre-average of a scalar can be defined as:

$$
\tilde{\phi}=\frac{1}{\bar{\rho}} \int_{c=0}^{1} \rho \phi(c) P(c) d c
$$

where the tilde indicates Favre-averaged variables and the overbar denotes Reynolds-averaged quantities. $P(c)$ indicates the probability density function of the RPV. A $\beta$-PDF shape is used in the current work to define the subgrid scale effects, since it is assumed that moderate levels of fluctuations occur for this case [17. The $\beta$-PDF is defined as:

$$
\beta\left(c, \tilde{c}, \widetilde{c^{\prime \prime 2}}\right)=\frac{c^{a-1}(1-c)^{b-1}}{\frac{\Gamma(a) \Gamma(b)}{\Gamma(a+b)}} \quad \text { with: } \quad a=\tilde{c}\left(\frac{\tilde{c}(1-\tilde{c})}{\widetilde{c^{\prime \prime 2}}}-1\right), b=\frac{1-\tilde{c}}{\tilde{c}} a
$$


with the constraint of $a, b>0$. From this constraint, the $\beta$-PDF is only valid for the variance of the reaction progress variable in the range $\widetilde{c^{\prime \prime 2}}<\tilde{c}(1-\tilde{c})$. In order to describe the entire $\left(\tilde{c}, \widetilde{c^{\prime \prime 2}}\right)$-space, a double $\delta$-PDF, which reproduces the behaviour of a $\beta$-PDF at high variance values, is used for combinations outside the valid range of the $\beta$-PDF [12].

The dimension of the resulting turbulent database is increased by one and can now be described by two controlling variables: the mean and the variance of the reaction progress variable. In the turbulent database, the variance of the reaction progress variable is tabulated at 25 discrete values. Since the $\beta$-function differs greatly in shape for small values of the variance a cubic subdivision of the grid points is chosen for the variance, distributing the points between zero and 0.25 [17]:

$$
\widetilde{c^{\prime \prime 2}}(j)=0.25\left(\frac{j}{n_{\widetilde{c^{\prime \prime 2}}}-1}\right)^{2} \quad\left(j=1, \ldots, n_{\widetilde{c^{\prime \prime 2}}}\right)
$$

The two-dimensional database is visualized in Fig. 5 on basis of the source term of the reaction progress variable. The laminar conditions correspond to a variance of zero. An increased value of the variance can be understood as an increased turbulence level. The effect of an increased turbulence intensity is similar to the effect of increased heat losses: the maximum value of the source term is reduced, slowing down the chemical reaction rates. Additionally, the source term is distributed over a wider range due to a smearing effect that also reduces the gradients of the source term.

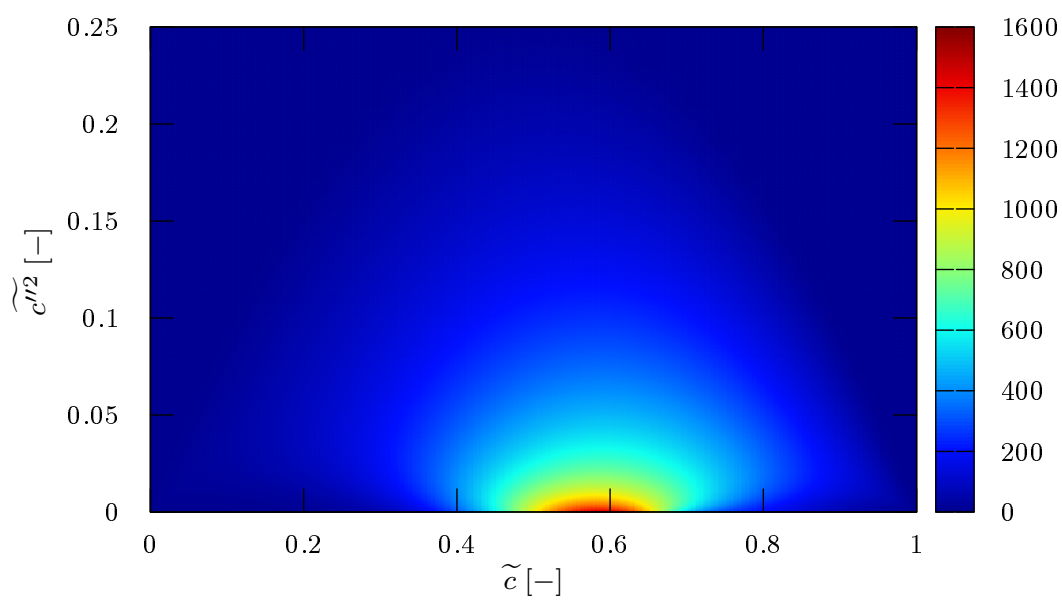

Figure 5: Source term of reaction progress variable $S_{c}\left[\frac{\mathrm{kg}}{\mathrm{m}^{3} \mathrm{~s}}\right]$ as function of mean and variance of RPV.

For non-adiabatic calculations, the chemical evolution in the laminar database depends not only on the reaction progress variable, but also on the enthalpy scalar $i$. If the scalars are linearly independent and not correlated due to the turbulent flow field, statistically independent fields can be assumed and a single scalar PDF can be employed for each variable. In general, only weak dependency is expected for enthalpy and RPV as the enthalpy is only changing due to heat losses which are independent of the RPV. Furthermore, heat loss occurs mainly in regions in which the RPV already equals unity and the fluctuations are zero. Additionally, statistical correlations caused by the geometry of the domain or physically allowed values are eliminated by the normalization of the variables. Therefore, the statistical correlations between the normalized variables 
are assumed to be negligible and the averaging process is done using a factorized joint PDF approach:

$$
\tilde{\phi}=\frac{1}{\bar{\rho}} \int_{c=0}^{1} \int_{i=0}^{1} \rho \phi(c, i) P(c) P(i) d i d c
$$

Due to the almost linear dependency of the species mass fractions and temperature on the enthalpy scalar $i$, turbulent fluctuations in $i$ are assumed to have only a small effect 37 and a $\delta$-PDF is employed for the enthalpy. The main advantage of using the $\delta$-PDF is that it only depends on the mean of the enthalpy scalar and higher moments do not need to be computed.

\subsection{Controlling variables}

The proposed combustion model is based on a reaction progress variable approach and a single scalar $c$ is used to describe the state of reaction. For turbulent flow calculations, the mean of the reaction progress $\tilde{c}$ and its first moment, the variance $\widetilde{c}{ }^{2}$, need to be determined to define the $\beta$-PDF. The following transport equations are solved for these two variables:

$$
\begin{gathered}
\bar{\rho} \frac{\partial \tilde{c}}{\partial t}+\bar{\rho} \tilde{\boldsymbol{u}} \cdot \nabla \tilde{c}=\nabla \cdot\left[\left(\bar{\rho} D+D_{t}\right) \nabla \tilde{c}\right]+\tilde{S}_{c} \\
\bar{\rho} \frac{\partial \widetilde{c " 2}}{\partial t}+\bar{\rho} \tilde{\boldsymbol{u}} \cdot \nabla \widetilde{c " 2}=\nabla \cdot\left[\left(\bar{\rho} D+D_{t}\right) \nabla \widetilde{c " 2}\right]+2\left(\widetilde{S_{c} c}-\tilde{S}_{c} \tilde{c}\right)+\bar{P}_{k}+\bar{D}_{k}
\end{gathered}
$$

Even though the formal description of the equations is the same in RANS and LES, time-averaging is applied for RANS, while spatial-filtering is employed for LES. The reaction source term $\tilde{S}_{c}$ is defined, analogous to the definition of the reaction progress variable itself, as the normalized sum of the weighted production rates of each species:

$$
S_{c}=\frac{\sum_{k=1}^{K} b_{k} \dot{\omega}_{k} W_{k}}{\eta^{b}-\eta^{u}}
$$

The source term is calculated in a pre-processing step and tabulated in the thermo-chemical database as function of the controlling variables. The laminar diffusion coefficient $D$ is determined based on the unity Lewis number assumption, as discussed in section 2.1.1. The turbulent diffusion coefficient $D_{t}$ originated from the unclosed term of the averaging or filtering operation is modelled using the eddy diffusivity hypothesis:

$$
D=\frac{\lambda}{\bar{\rho} c_{p}}, \quad D_{t}=\frac{\mu_{t}}{S c_{t}}
$$

where $\mu_{t}=\bar{\rho} \nu_{t}$ is the turbulent viscosity and $S c_{t}$ is the turbulent Schmidt number and is set to $S c_{t}=0.9$ for the current case. $\bar{P}_{k}$ and $\bar{D}_{k}$ are the production and dissipation terms, respectively. Their modelling is essentially different for RANS and LES. In RANS the production and dissipation terms are given by [12]:

$$
\bar{P}_{k}=2 \bar{\rho} \frac{\nu_{t}}{S c_{t}}|\nabla \tilde{c}|^{2}, \quad \bar{D}_{k}=-2 \bar{\rho} \frac{\varepsilon}{k} \widetilde{c}^{2}
$$

where the turbulent kinetic energy $k$ and the turbulent dissipation rate $\varepsilon$ as well as the turbulent viscosity $\nu_{t}$ are computed by the use of a turbulence model. In the LES approach, the formulation proposed by Domingo et al. 17 is followed. The subgrid scale part of the scalar dissipation rate is modelled with the linear relaxation hypothesis and defining a filter width $\Delta$, the production and dissipation terms are given by:

$$
\bar{P}_{k}=2 \bar{\rho} \frac{\nu_{t}}{S c_{t}}|\nabla \tilde{c}|^{2}, \quad \bar{D}_{k}=-2 \bar{\rho} \frac{\nu_{t}}{\Delta^{2} S c_{t}} \widetilde{c}^{\pi 2}
$$

In the LES, the turbulent viscosity is computed by the subgrid-scale model. The effect of the variance in the LES is to thicken the flame front over the LES grid and wrinkling the flame due to the interaction between the reaction zone and the subgrid scale vortices. 
To couple the thermo-chemical database to the numerical simulation in the non-adiabatic case, the mean value of the normalized enthalpy scalar $\tilde{i}$ is required. However, the enthalpy scalar is only used to access the correct entry in the database and it is computed based on the non-normalized enthalpy presented in the next section (Eq. 18). Therefore, no additional transport equation is required for the enthalpy scalar itself.

\subsection{Governing equations of fluid dynamics}

For the sake of simplicity, the governing equations in RANS and LES will be written again in the same form. However, note that time-averaging is applied in RANS, while spatial-filtering is employed in LES. The time-averaged (RANS) or filtered (LES) equations governing the reacting flow field are the continuity, momentum and enthalpy equations:

$$
\begin{gathered}
\frac{\partial \bar{\rho}}{\partial t}+\nabla \cdot(\bar{\rho} \tilde{\boldsymbol{u}})=0 \\
\bar{\rho} \frac{\partial \tilde{\boldsymbol{u}}}{\partial t}+\bar{\rho} \tilde{\boldsymbol{u}} \cdot \nabla \tilde{\boldsymbol{u}}=-\nabla \bar{p}+\nabla \cdot \boldsymbol{\tau}+\nabla \cdot \boldsymbol{\tau}^{*} \\
\bar{\rho} \frac{\partial \tilde{h}}{\partial t}+\bar{\rho} \tilde{\boldsymbol{u}} \cdot \nabla \tilde{h}=\nabla \cdot\left(\frac{\lambda}{c_{p}} \nabla \tilde{h}\right)+\nabla \cdot h^{*}
\end{gathered}
$$

where the physical variables are represented by the standard notation and the superscript $*$ stands for the unclosed terms coming from the filtering or averaging operation. The heat production due to viscous forces is neglected in the enthalpy equation. The unclosed term in the momentum equation is modelled using the Wall-Adapting Local Eddy-viscosity model (WALE) 38] in LES, while the $k-\omega$-SST [39] is used in RANS. For both RANS and LES, the unresolved heat flux is modelled using a gradient diffusion approach [40]:

$$
h^{*}=\frac{\mu_{t}}{S c_{t}} \nabla \tilde{h}
$$

The enthalpy is defined as the sum of the sensible and chemical enthalpy, which is defined for each species as:

$$
h_{k}=\int_{T_{0}}^{T} c_{p, k} d T+\Delta h_{k}^{0}
$$

This is the low-Mach approximation, neglecting kinetic energy. The enthalpy of the mixture is then obtained by a summation over the species:

$$
h=\sum_{k=1}^{K} Y_{k} h_{k}
$$

The temperature is linked to the enthalpy by a polynomial expression:

$$
h=\sum_{n=1}^{5} \frac{a_{n}}{n} T^{n}+a_{6}
$$

in which the $a_{n}$ represent the NASA coefficients [4]. The NASA coefficients have been designed to efficiently describe thermodynamic data. The coefficients depend on the local composition and are tabulated in the database for two different temperature ranges as a function of the controlling variables. The temperature is computed by inversion of Eq. 22. In practice, an iterative procedure based on a Newtons method is used to obtain the temperature implicitly. In addition to the thermodynamic coefficients and the reaction source term, mixture-averaged transport properties are tabulated for the thermal conductivity and the laminar viscosity. For the tabulation of these properties, deviations of the local CFD temperature from the flamelet temperature are neglected. 


\section{One-dimensional laminar premixed flame}

In a first attempt to validate the modelling approach and the chemistry tabulation described in the previous section, a one-dimensional laminar premixed flame calculation is carried out. The flamelet, that has been computed with PREMIX and is used for the chemistry tabulation (see section 2.1), serves as a reference.

A two-dimensional domain is employed with symmetrical boundary conditions at the top and bottom end. The domain length is $l=2 \mathrm{~cm}$, which is slightly longer than the PREMIX domain. The element size is determined based on the laminar flame thickness, which is obtained based on the PREMIX results using the following expression [36]:

$$
\delta_{l}^{0}=\frac{T_{b}-T_{u}}{\max \left(\left|\frac{\partial T}{\partial x}\right|\right)}=4.14 \times 10^{-4} \mathrm{~m}
$$

It is found that 8 grid points placed inside the flame front are sufficient to describe accurately the temperature gradients. The mesh is created under this hypothesis and results in a total number of 400 elements. Contrary to the PREMIX computation, where most of the grid points are located in the flame front, the Alya mesh has a uniform spacing. The laminar flame speed obtained from the PREMIX calculation is used as inlet velocity at the cold boundary in the Alya computation. The same database as in the turbulent case is used, since by using $\widetilde{c^{\prime \prime 2}}=0$, the laminar behaviour is retained.

The profiles of the temperature as well as some representative species are presented in Fig. 6 and compared to the reference solution. The results are in good agreement with the reference solution. Only the temperature at the hot boundary is slightly overpredicted in the Alya computation. The chemical composition is linked to the reaction progress variable and if the profile of the reaction progress variable is correctly computed, all species will also exhibit the correct behaviour. Consequently, the profiles of the mass fractions are computed accurately for all species. Even intermediate species like HCO, which only occur in the very thin flame front, are well predicted. This leads to the conclusion that the tabulation operation as well as the coupling procedure to access the chemical database is accurate.
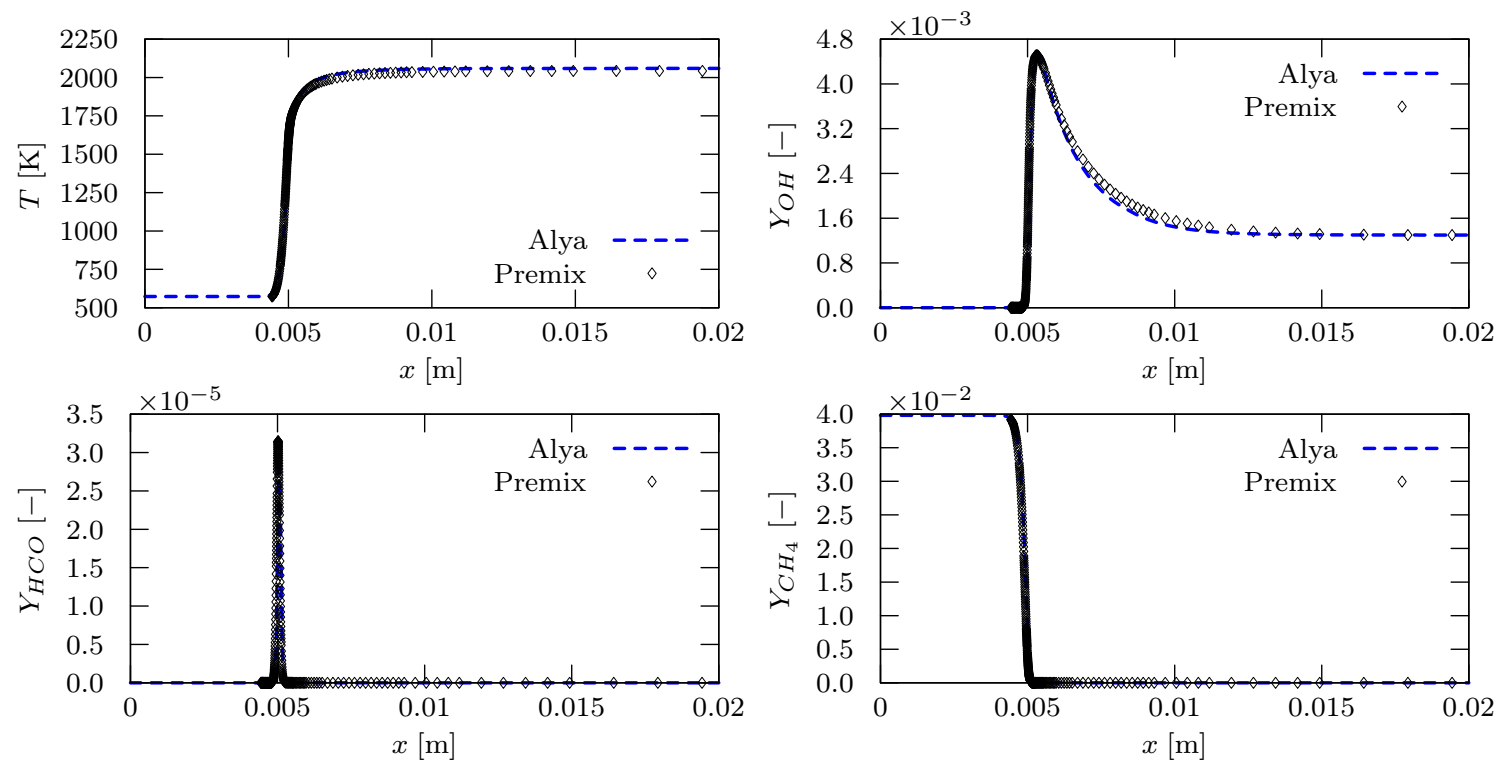

Figure 6: Comparison of one-dimensional laminar premixed flame results for the temperature and selected species. 


\section{Turbulent premixed jet flame}

This section focuses on the comparison of the numerical simulations, using the proposed combustion model, with the experimental data of a turbulent premixed jet flame. After a brief introduction of the test case, the simulation setup is given for RANS and LES. Finally the results are presented and discussed.

\subsection{Test case description}

The test case that is used for validation of the turbulent combustion model corresponds to an experimental facility at the German Aerospace Center (DLR). The operating point that is used in the current case is part of a test series of measurements conducted by Lammel et al. 23. The combustor was designed to investigate the flameless oxidation $\left(\mathrm{FLOX}^{\circledR}\right)$ regime and its application to gas turbines. It is a premixed turbulent jet flame confined in a rectangular combustion chamber and operated at atmospheric pressure. A methane/air mixture at an equivalence ratio of 0.71 is injected into the combustion chamber through a circular pipe. The jet nozzle is positioned off-centred to achieve a pronounced lateral recirculation zone and the flame is stabilized by the recirculation of hot combustion products. At the inlet, the mixture is preheated up to 573 $\mathrm{K}$ and injected with an inlet velocity of $v_{\text {in }}=90 \mathrm{~m} / \mathrm{s}$. The walls of the combustion chamber are made of quartz glass to allow for optical access required for laser-based measurements. Due to the combustor design, conductive heat losses to the wall are significant and a distinctive feature of the test case.

A sketch of the geometry is given in Fig. 77. Small geometrical details are removed in order to improve the meshing procedure. To reduce the computational requirements, the computational domain is shortened and extends up to 40 nozzle diameters $d$. As the flame is short respect to the combustor, the outlet of the computational domain is still located sufficiently far away from the region of interest. For the same reason, the length of the inlet pipe is also reduced.

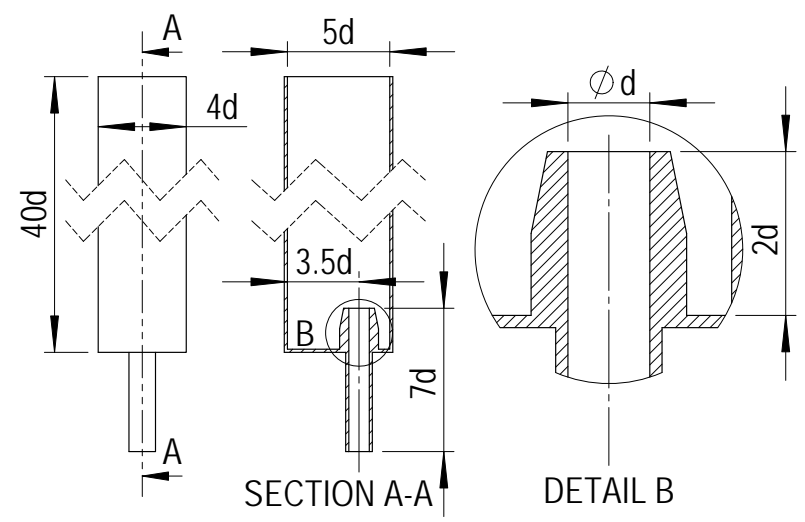

Figure 7: Sketch of the confined jet flame geometry as used in the CFD simulation.

\subsection{Simulation setup}

In this section, the setup for RANS and LES simulations is presented. A part of the inlet pipe is included in the domain in order to allow for a natural development of the turbulent flow field before entering the combustion chamber and to reduce the influence of the boundary condition at the inlet. The inlet boundary conditions are set with top-hat profiles for temperature, progress variable and velocity. A random profile of white noise at $10 \%$ of turbulent intensity is superimposed to the velocity profile for LES to speed up the development of real turbulence. No-slip conditions are set for the velocity at the walls in the LES, while Reichardt's law-of-the-wall 42 is used for RANS, which has the advantage of describing all different regions of the turbulent boundary layer. The domain has been extended in the streamwise direction coarsening the grid to allow for the formation of a sponge layer and outflow conditions are applied for all variables at the outlet. The test case is characterized by considerable heat losses at the walls, but no measurements of the wall temperature are available, so the exact heat transfer conditions are unknown. Proch et al.26] 
have chosen a wall temperature of $T_{w}=1000 \mathrm{~K}$ based on the ageing behaviour of the quartz glass walls and claimed that the simulation results only showed a low sensitivity to this value. For the non-adiabatic simulations presented in this paper, isothermal wall boundary conditions are used for the enthalpy equation corresponding to a wall temperature of $T_{w}=1000 \mathrm{~K}$. However, in our simulations, a strong sensitivity of the wall temperature on temperature profiles is found. For further improvement and to take into account spatial variations, a conjugate heat transfer approach will be considered in the future. For the adiabatic case, a zero gradient condition for the enthalpy is applied.

The spatial discretization is based on the Finite Element method using the Variational Multiscale Stabilization technique 21. A second-order Crank-Nicholson time integration scheme is used for LES, while a first order implicit backward Euler is used to obtain steady state solutions for RANS. Several grids with different resolution have been investigated to evaluate the dependency of the results with the mesh size. For the sake of brevity, only the results with the finest grid will be presented here. The mesh is unstructured and consists of about $5.0 \mathrm{M}$ tetrahedral elements. The cell size in the inlet pipe and the flame region is about $0.08 \mathrm{~d}$ and gradually coarsens in the downstream regions towards the outlet. The same mesh is used for both LES and RANS simulations. An unsteady-RANS approach with a high Courant-Friedrichs-Lewy (CFL) number is applied to obtain the steady state fields in the RANS simulation. Time-averaged fields are presented, even though the time dependent fluctuations are very minor. The LES is run at a maximum CFL number of about 6 . To remove the influence of initialization effects, the averaging process is started after 1.2 flow through times based on the averaged streamwise velocity in the domain. The flow was then time-averaged for a total of 4 flow through times based on the domain-averaged velocity, which corresponds to about 45 flow through times based on bulk velocity.

\subsection{Results}

This section addresses the comparison of the numerical simulations for RANS and LES using the proposed combustion model with the experimental data from Lammel et al. 23. The main flow features can be identified from the instantaneous fields presented in Fig. 8. The measured axial velocity is compared to the velocity predicted by the LES simulation. The lateral recirculation zone created by the off-centre positioning of the jet nozzle can be identified. This recirculation is well known to provide flame stability by bringing back upstream the hot products of combustion [43. The local structures of the velocity observed in the experiments are accurately reproduced by the LES fields. These structures are caused by shear layer instabilities and also affect the temperature fields. These unsteady structures enhance the mixing process between hot products and cold reactants and, therefore, play an important role in the convective heat transfer.

A quantitative evaluation of the prediction capabilities of the proposed model for both RANS and LES is obtained by the comparison of axial profiles located at different locations: one, two, four, ten and fifteen nozzle diameters downstream of the jet exit. The uncertainties of the measurements are estimated to be 2.3 $\mathrm{m} / \mathrm{s}$ for the velocity measurements and about $5 \%$ for the measured temperature and species distribution [23]. The respective values are added to the profiles. In Fig. 9, the time averaged results of the LES simulation are presented for different heat loss modelling approaches. In the first approach, heat loss is taken into account by imposing isothermal boundary conditions for the enthalpy equation but the effect of the heat loss on the chemical reactions is neglected. In the second approach, the effect of heat loss on the chemical reactions is taken into account by the introduction of an additional dimension in the chemistry tabulation (see section 2.1.3). The predicted axial velocity is in agreement with the measurements at all axial locations and only a small influence of the heat loss modelling approach is observed. The correct prediction of the transversal velocity is more difficult to capture due to the small absolute values and requires longer averaging times. Next to the relatively higher uncertainty in the measurements, there is an influence of the timeaverage period in the results at downstream locations, which are characterized by the slow scales. However, the flow features are correctly predicted at most locations with minor differences among the cases. The temperature profiles evidence the strong influence of heat loss in this burner configuration that contributes to the reduction of the burning rates and peak temperatures. The reduction of the peak temperature is already well predicted by the modelling approach in which the effect of the heat loss on the chemical reactions is neglected. It is found that the flame length is more accurately predicted taking into account the reduction 


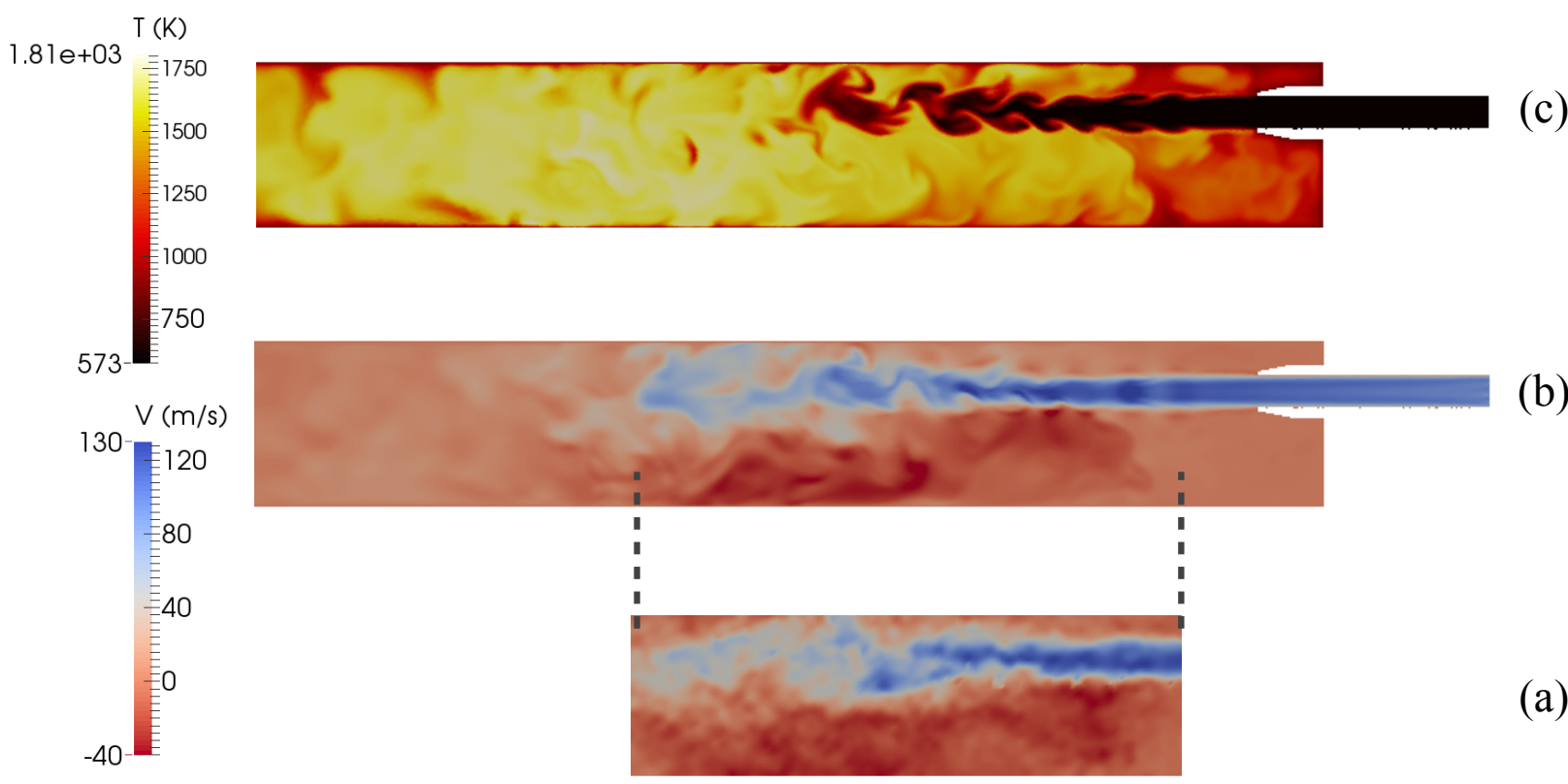

Figure 8: Instantaneous fields of axial velocity (a) experiments and (b) simulation; and temperature (c) simulation.

of the reaction rates by heat losses. The temperature in the recirculation zone is slightly underpredicted at some locations by the simulation. This is most likely caused by the thermal boundary condition at the walls, which is constant along the entire combustor.

The corresponding root-mean-square (RMS) values for the LES are presented in Fig. 10, The fluctuations at the shear layer are predicted by the LES even though the magnitude is not captured accurately at all locations. The temperature fluctuations show the correct trend as compared to the experimental data, although the RMS values are underpredicted at the last measurement location due to the underprediction of the flame length. For both heat loss modelling approaches, the fluctuations in the recirculation zone at locations close to the jet nozzle are slightly underpredicted. In general, the predicted flow field is expected to improve if the mesh is further refined.

The same simulations are performed using the RANS formulation and the results are shown in Fig. 11 for comparison. For completes, the results using adiabatic thermal boundary conditions are presented as well and emphasize the strong effect of heat loss in this test case. Both, axial and transversal velocity, are accurately predicted at all measurement locations. Only the transversal velocity in the recirculation zone is slightly overpredicted. Almost no differences in the velocity fields are observed for the different heat loss modelling approaches. For the non-adiabatic modelling approaches, the temperature is accurately captured in the recirculation zone. However, contrary to the LES, the flame length is only slightly affected by the different heat loss modelling approaches. This is caused by the underpredicted turbulence at the shear layer that induces low mixing rates. The low level of mixing leads to an underprediction of the convective heat transfer and hence, a shorter flame.

In the proposed model, a reaction progress variable is used for the description of the chemistry. During the computation, the local mixture composition is only used to determine the transport and thermodynamic properties. However, in a post-processing step detailed information about the chemical composition can be obtained using the fields of the controlling variables. Profiles of some major species mole fractions from the LES calculations are presented and compared to the experimental data in Fig. 12. Only a small effect of the heat losses in the chemistry is observed for the locations close to the jet nozzle. However, for the downstream profile, significant differences in the composition occur due to the reduced chemical time scales for the case in which the chemistry is affected by heat loss. 

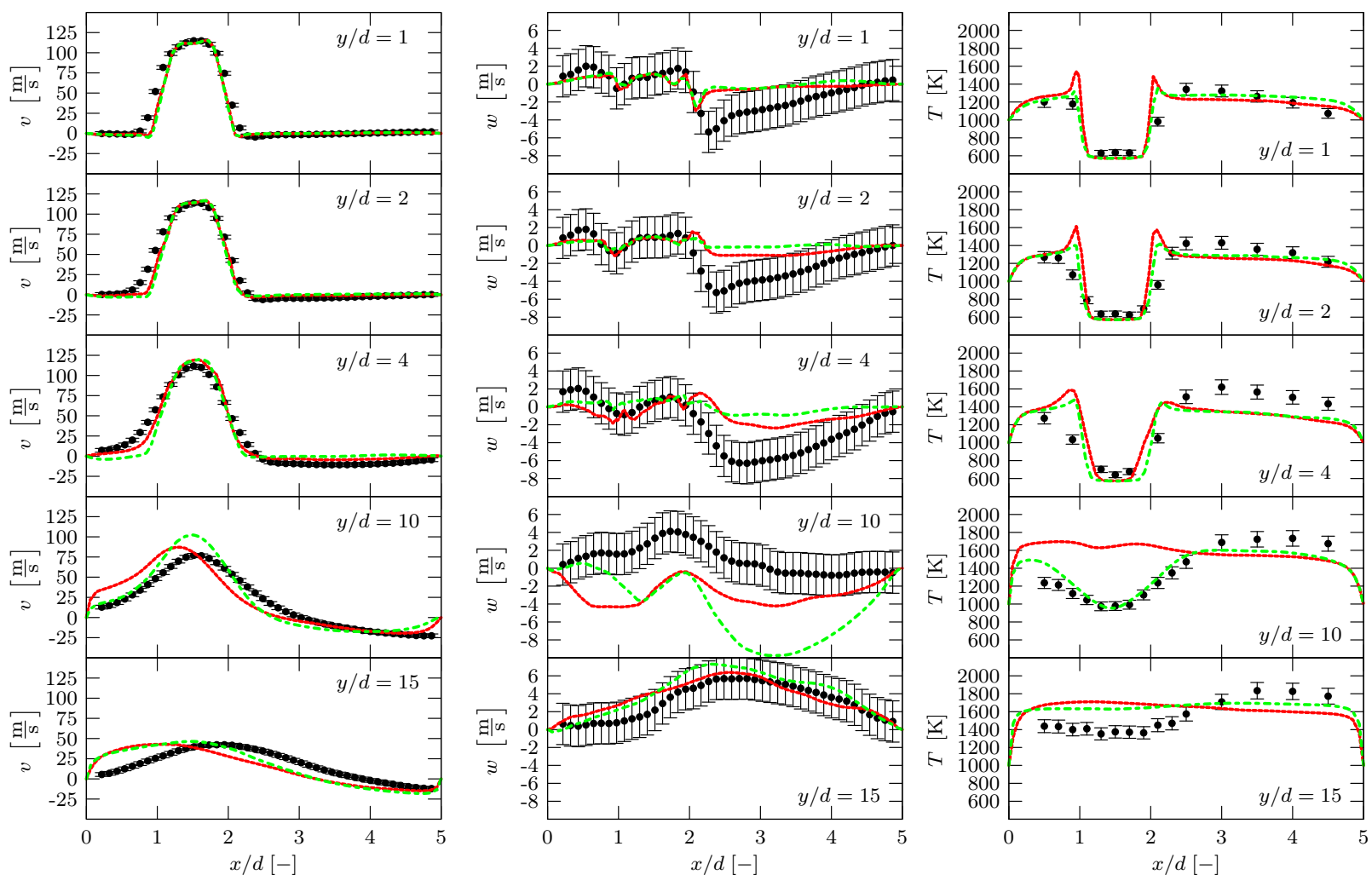

Figure 9: Profiles of time-averaged axial and transversal velocity and temperature for the LES simulations (dots: experiments, red: non-adiabatic, green: non-adiabatic with heat loss in chemistry).

\section{Conclusions and future work}

A turbulent combustion model for premixed combustion is presented with application for RANS and LES. The model is based on the tabulation of chemistry using laminar premixed flamelets and a reaction progress variable is introduced to describe the state of reaction. An optimized choice of the RPV is obtained by application of the CSP method. The proposed model is tested in the framework of a CFD code that is specifically designed for large scale computations. The results for a laminar one-dimensional premixed flame calculation show excellent agreement with the detailed chemistry simulation and prove the suitability of the chemistry tabulation based on the RPV for an accurate description of the chemical kinetics at reduced computational cost.

Results are presented for a turbulent premixed jet flame confined in a rectangular combustion chamber. The main flow features are predicted correctly in both RANS and LES. The coherent structures due to the free shear layer are reproduced by the LES. Different approaches for the modelling of heat losses are presented and compared with the experimental data. It is shown that the influence of heat loss on the chemical reactions can be neglected in order to predict the peak temperatures but needs to be taken into account for a correct prediction of the flame length. The flame length is not very accurately computed in the RANS simulations due to the underprediction of the turbulent mixing and the convective heat transfer in the shear layer. The LES fields show a good overall agreement with the experimental data and better predictions are expected with more appropriate thermal boundary conditions at the walls. 

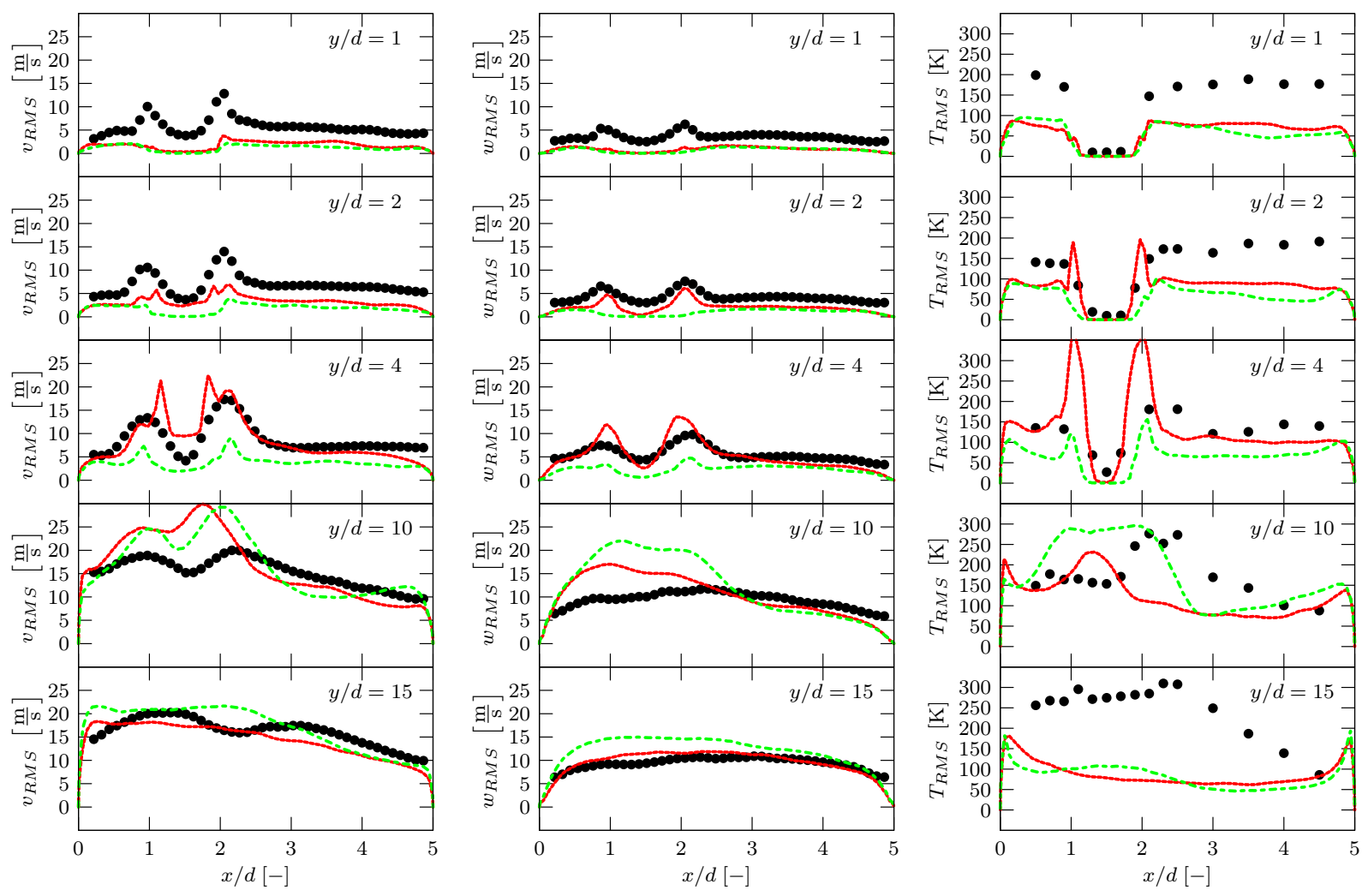

Figure 10: Profiles of RMS values for axial and transversal velocity and temperature for the LES simulations (dots: experiments, red: non-adiabatic, green: non-adiabatic with heat loss in chemistry).

\section{Acknowledgements}

The research leading to these results has received funding through the People Programme (Marie Curie Actions) of the European Union's Seventh Framework Programme (FP7, 2007-2013) under the grant agreement No. FP7-290042 for the project COPA-GT. The authors thankfully acknowledge the computer resources, technical expertise and assistance provided by the Red Española de Supercomputación (RES). Finally, the authors would like to thank O. Lammel for kindly providing the data for comparison.

\section{References}

[1] A. E. Khalil, A. K. Gupta, Fuel flexible distributed combustion for efficient and clean gas turbine engines, Applied Energy 109 (2013) $267-274$.

[2] International Energy Agency, Key world energy statistics, 2013. URL: http://www.iea.org/publications/ freepublications/publication/KeyWorld2013.pdf

[3] U. Stopper, W. Meier, R. Sadanandan, M. Sthr, M. Aigner, G. Bulat, Experimental study of industrial gas turbine flames including quantification of pressure influence on flow field, fuel/air premixing and flame shape, Combust. Flame 160 (2013) $2103-2118$

[4] V. Raman, H. Pitsch, A consistent les/filtered-density function formulation for the simulation of turbulent flames with detailed chemistry, Proceedings of the Combustion Institute 31 (2007) 1711-1719.

[5] C. Duwig, K.-J. Nogenmyr, C. ki Chan, M. J. Dunn, Large eddy simulations of a piloted lean premix jet flame using finite-rate chemistry, Combustion Theory and Modelling 15 (2011) 537-568.

[6] O. Gicquel, N. Darabiha, D. Thevenin, Laminar premixed hydrogen/air counterflow flame simulations using flame prolongation of ILDM with differential diffusion, Proceedings of the Combustion Institute 28 (2000) $1901-1908$.

[7] J. van Oijen, F. Lammers, L. de Goey, Modeling of complex premixed burner systems by using flamelet-generated manifolds, Combustion and Flame 127 (2001) $2124-2134$. 

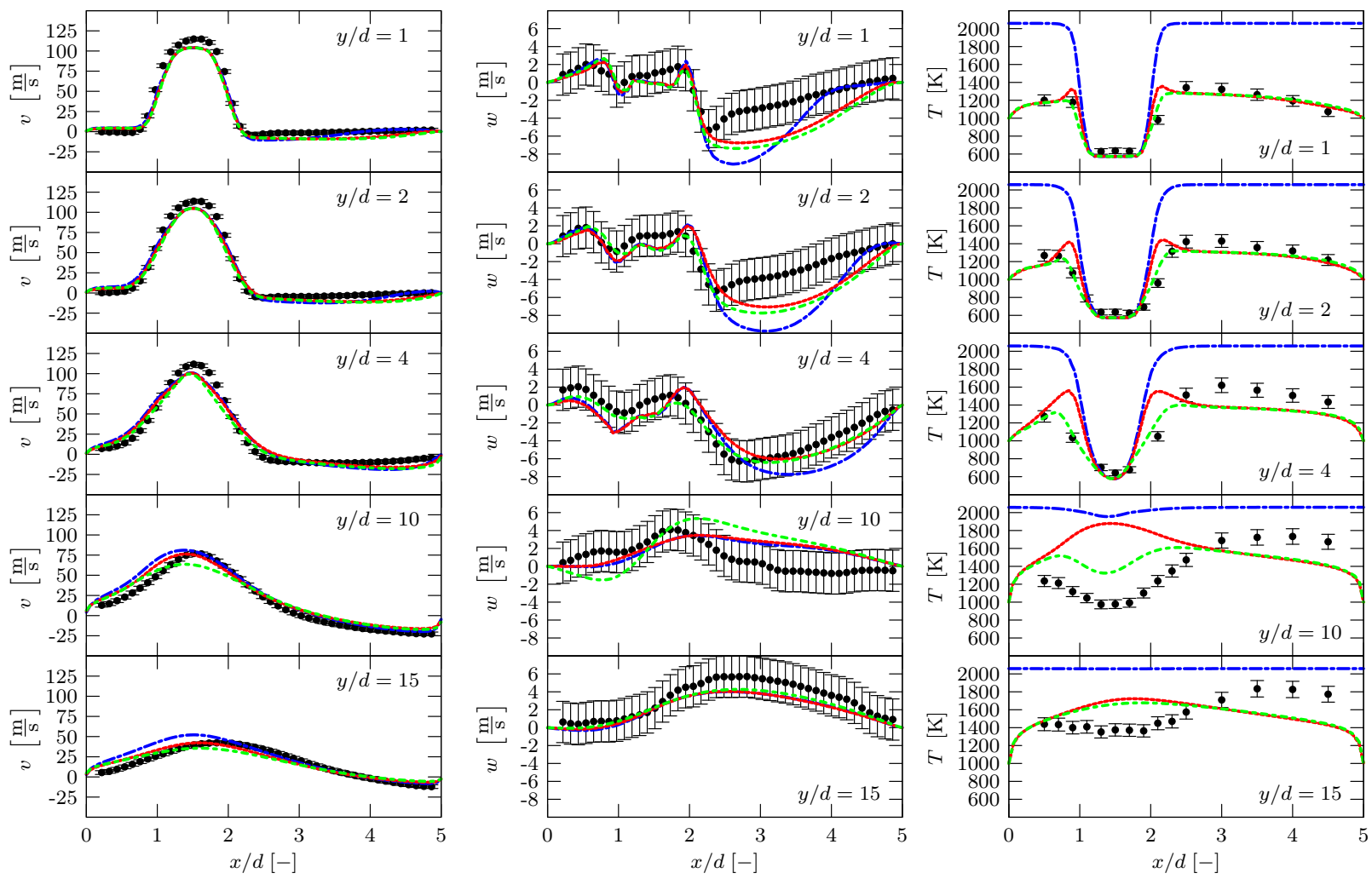

Figure 11: Profiles of time-averaged axial and transversal velocity and temperature for the RANS simulations (dots: experiments, blue: adiabatic, red: non-adiabatic, green: non-adiabatic with heat loss in chemistry).

[8] B. Fiorina, O. Gicquel, L. Versvich, S. Carpentier, N. Darabiha, Premixed turbulent combustion modeling using tabulated chemistry and pdf, Proc. Combust. Inst. 30 (2005) 867-874.

[9] M. Ihme, C. Cha, H. Pitsch, Prediction of local extinction and re-ignition effects in non-premixed turbulent combustion by a flamelet/progress variable approach, Proc. Combust. Inst. 30 (2005) 793-800.

[10] C. Pierce, P. Moin, Progress-variable approach for large-eddy simulation of non-premixed turbulent combustion, J. Fluid Mech. 504 (2004) 73-79.

[11] U. Maas, S. Pope, Simplifying chemical kinetics: Intrinsic low-dimensional manifolds in composition space, Proc. Combust. Inst. 24 (1992) 103-112.

[12] M. Derksen, On the influence of steam on combustion, Ph.D. thesis, University of Twente, 2005.

[13] A. Massias, D. Diamantis, E. Mastorakos, D. Goussis, An algorithm for the construction of global reduced mechanisms with CSP data, Combust. Flame 117 (1999) $685-708$.

[14] B. Albrecht, Reactor modeling and process analysis for partial oxidation of natural gas, $\mathrm{Ph} . \mathrm{D}$. thesis, University of Twente, 2004.

[15] J. van Oijen, L. de Goey, Modelling of premixed laminar flames using flamelet-generated manifolds, Combust. Sci. Technol. 161 (2000) 113-137.

[16] A. Vreman, B. Albrecht, J. van Oijen, L. de Goey, R. Bastiaans, Premixed and nonpremixed generated manifolds in large-eddy simulation of Sandia flame D and F, Combust. Flame 153 (2008) $394-416$.

[17] P. Domingo, L. Vervisch, S. Payet, R. Hauguel, Dns of a premixed turbulent v flame and les of a ducted flame using a fsd-pdf subgrid scale closure with fpi-tabulated chemistry, Combust. Flame 143 (2005) 566586.

[18] M. Ihme, L. Shunn, J. Zhang, Regularization of reaction progress variable for application to flamelet-based combustion models, Journal of Computational Physics 231 (2012) 7715 - 7721

[19] Y.-S. Niu, L. Vervisch, P. D. Tao, An optimization-based approach to detailed chemistry tabulation: Automated progress variable definition, Combustion and Flame 160 (2013) 776 - 785

[20] BSC-CNS, Alya multiphysics code, 2014. URL: http://www.bsc.es/computer-applications/alya-system

[21] G. Houzeaux, J. Principe, A variational subgrid scale model for transient incompressible flows, Int. J. Comput. Fluid D. 22 (2008) 135-152.

[22] M. Vazquez, G. Houzeaux, S. Koric, A. Artigues, J. Aguado-Sierra, R. Aris, D. Mira, H. Calmet, F. Cucchietti, H. Owen, A. Taha, J. M. Cela, Alya: Towards exascale for engineering simulation codes, in: International Supercomputing 

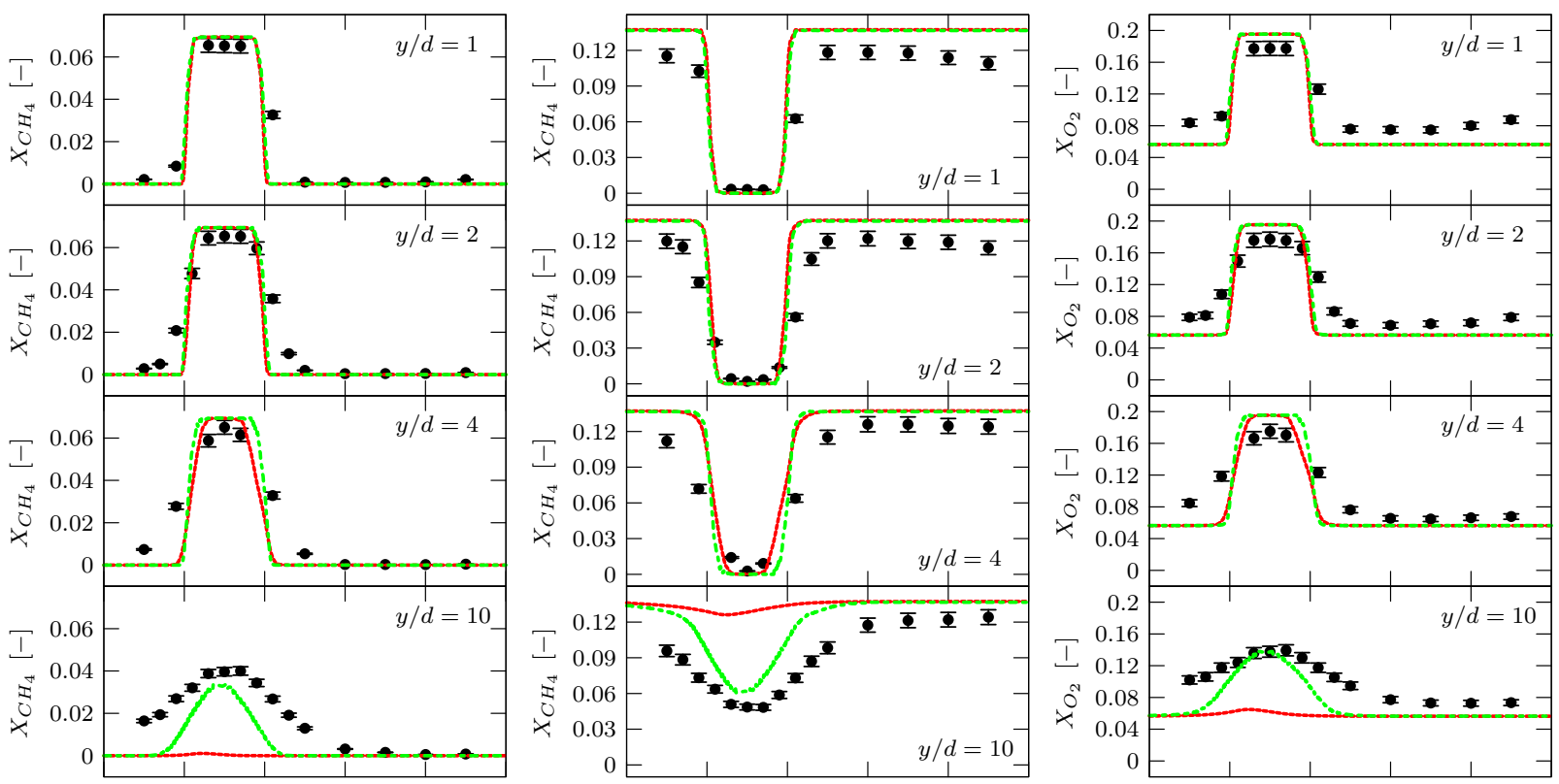

Figure 12: Profiles of major species for the LES simulations (dots: experiments, red: non-adiabatic, green: non-adiabatic with heat loss in chemistry).

Conference 2014, arXiv:1404.4881[physics.comp-ph], 2014.

[23] O. Lammel, M. Stöhr, P. Kutne, C. Dem, W. Meier, M. Aigner, Experimental analysis of confined jet flames by laser measurement techniques, J. Eng. Gas Turbines Power 134 (2012).

[24] A. Donini, S. Martin, R. Bastiaans, J. van Oijen, L. de Goey, Numerical simulations of a premixed turbulent confined jet flame using the flamelet generated manifold approach with heat loss inclusion, in: Proc. ASME Turbo Expo, San Antonio, Texas, 2013.

[25] A. Fancello, L. Panek, O. Lammel, W. Krebs, R. Bastiaans, L. de Goey, Turbulent combustion modeling using flameletgenerated manifolds for gas turbine applications in openfoam, in: Proceedings of the ASME Turbo Expo 2014, Düsseldorf, Germany, 2014.

[26] F. Proch, A. Kempf, Modeling heat loss effects in the large eddy simulation of a model gas turbine combustor with premixed flamelet generated manifolds, Proceedings of the Combustion Institute 35 (2015) $3337-3345$.

[27] R. Kee, G. Dixon-Lewis, J. Warnatz, M. Coltrin, J. Miller, A Fortran Computer Code Package for the Evaluation of Gas-Phase Multicomponent Transport Properties, Technical Report SAND86-8246, Sandia National Laboratories, 1986.

[28] R. Kee, F. Rupley, J. Miller, M. Coltrin, J. Grcar, E. Meeks, H. Moffat, A. Lutz, G. Dixon-Lewisa, M. Smooke, J. Warnatz, G. Evans, R. Larson, R. Mitchell, L. Petzold, W. Reynolds, M. Caracotsios, W. Stewart, P. Glarborg, C. Wang, O. Adigun, Chemkin collection, release 3.6 (2000).

[29] G. Smith, D. Golden, M. Frenklach, N. Moriarty, B. Eiteneer, M. Goldenberg, C. T. Bowman, R. Hanson, S. Song, W. Gardiner, V. Lissianski, Z. Qin, GRI-Mech 3.0, 1999. URL: http://www.me.berkeley.edu/gri-mech/

[30] A. Donini, R. Bastiaans, J. van Oijen, L. de Goey, Differential diffusion effects inclusion with flamelet generated manifold for the modeling of stratified premixed cooled flames, Proceedings of the Combustion Institute 35 (2015) $831-837$.

[31] J. J. Louis, J. B. Kok, S. A. Klein, Modeling and measurements of a 16-kw turbulent nonadiabatic syngas diffusion flame in a cooled cylindrical combustion chamber, Combustion and Flame 125 (2001) $1012-1031$.

[32] B. de Jager, J. Kok, G. Skevis, The effects of water addition on pollutant formation from lpp gas turbine combustors, Proceedings of the Combustion Institute 31 (2007) 3123-3130. Proceedings of the 31st International Symposium on Combustion, Heidelberg, Germany, August 6-11, 2006.

[33] B. de Jager, J. Kok, Modeling of turbulent combustion of lean premixed prevaporized liquid fuel using the cfi combustion model, in: Proc. ASME Turbo Expo, Barcelona, Spain, 2006.

[34] D. Cecere, E. Giacomazzi, F. Picchia, N. Arcidiacono, F. Donato, R. Verzicco, A non-adiabatic flamelet progressvariable approach for les of turbulent premixed flames, Flow, Turbulence and Combustion 86 (2011) 667-688.

[35] B. Fiorina, R. Baron, O. Gicquel, D. Thevenin, S. Carpentier, N. Darabiha, Modelling non-adiabatic partially premixed flames using flame-prolongation of ildm, Combustion Theory and Modelling 7 (2003) 449-470.

[36] T. Poinsot, D. Veynante, Theoretical and Numerical Combustion, Ed. Edwards, 3rd Edition, 2012.

[37] J. Kok, J. Louis, J. Yu, The irst model for turbulent premixed non-adiabatic methane flames, Combustion Science and Technology 149 (1999) 225-247. 
[38] F. Nicoud, F. Ducros, Subgrid-scale stress modelling based on the square of the velocity gradient, Flow Turb. Combust. 62 (1999) 183-200.

[39] F. Menter, Two-equation eddy-viscosity turbulence models for engineering applications, AIAA J. 32 (1994) $1598-1605$.

[40] D. Mira, X. Jiang, C. Moulinec, D. Emerson, Numerical assessment of subgrid scale models for scalar transport in large-eddy simulations of hydrogen-enriched fuels, Int. J. Hydrogen Energy 39 (2014) 7173-7189.

[41] B. McBride, S. Gordon, M. Reno, Coefficients for calculating thermodynamic and transport properties of individual species, Technical Report, NASA Lewis Research Center; Cleveland, OH, United States, 1993.

[42] H. Reichardt, Vollstndige darstellung der turbulenten geschwindigkeitsverteilung in glatten leitungen, ZAMM - Journal of Applied Mathematics and Mechanics / Zeitschrift fr Angewandte Mathematik und Mechanik 31 (1951) 208-219.

[43] V. K. Arghode, A. K. Gupta, Effect of flow field for colorless distributed combustion (cdc) for gas turbine combustion, Applied Energy 87 (2010) 1631 - 1640. 